\title{
Association between the CCR2-Val64Ile polymorphism and susceptibility to HIV-1 infection: a meta-analysis
}

\author{
DONG-LIN DING, SI-JIE LIU and HUAN-ZHANG ZHU
}

\begin{abstract}
The State Key Laboratory of Genetic Engineering, Institute of Genetics, School of Life Sciences, Fudan University, Shanghai 200433, P.R. China
\end{abstract}

Received August 5, 2010; Accepted November 29, 2010

DOI: $10.3892 / \mathrm{mmr} .2010 .400$

\begin{abstract}
Several studies have investigated whether the CCR2-Val64Ile polymorphism affects susceptibility to human immune deficiency virus type-1 (HIV-1), with inconclusive results. Here, we performed a meta-analysis of the literature aiming to clarify the relationship between the polymorphism of CCR2-Val64Ile and the risk of HIV-1 infection. Twelve studies with a total of 6,599 patients, including infants, were selected for inclusion in the analysis. Crude odds ratios (ORs) with $95 \%$ confidence intervals (CIs) were assessed after the collected data were pooled for analysis. The risk estimates (OR) of HIV-1 infection were calculated in a homozygote comparison $(\mathrm{OR}=1.10,95 \%$ CI $0.79-1.53)$, a heterozygote comparison $(\mathrm{OR}=0.98,95 \%$ CI $0.70-1.37)$, a dominant model $(\mathrm{OR}=1.06,95 \% \mathrm{CI} 0.77-1.47)$ and a recessive model $(\mathrm{OR}=0.98$, $95 \%$ CI $0.77-1.27$, by random effects model) from among the total population. Taking into account the effect of sample size, ethnicity and control population, further stratified analyses were performed. The results showed no statistically significant difference in any genetic model, with the exception of the subanalysis of mixed ethnicity (OR=0.33, 95\% CI 0.11-0.98) using heterozygote comparison. The meta-analysis clarified that the CCR2-Val64Ile polymorphism has no effect on susceptibility to HIV-1 infection in the total population.
\end{abstract}

\section{Introduction}

Nearly 40 million people have been infected by human immune deficiency virus type-1 (HIV-1) worldwide (http://data.unaids. org/pub/Report/2007/JC1299-PolicyBrief-GIPA_en.pdf). Almost 27 million people have died of acquired immunodeficiency syndrome (AIDS) caused by HIV-1 infection; an estimated 2 million people every year. HIV prevalence (http://

Correspondence to: Professor Huan-Zhang Zhu, The State Key Laboratory of Genetic Engineering, Institute of Genetics, School of Life Sciences, Fudan University, Shanghai 200433, P.R. China E-mail: hzzhu@fudan.edu.cn

Key words: CCR2-Val64Ile, polymorphism, human immune deficiency virus type-1, meta-analysis www.who.int/features/factfiles/hiv/facts/en/index3.html) can therefore be considered the greatest social, economic and health crisis of modern times (1).

Previous studies have reported that the polymorphisms of certain chemokines and chemokine receptors, including stromal cell derived factor-1, RANTES, chemokine receptor-2 (CCR2) and chemokine receptor-5, play important roles in HIV-1 pathogenesis and transmission (2-8). Among them, CCR2, which is located at band p21 of chromosome 3 and contains three exons distributed over $7 \mathrm{~kb}$ of the genomic sequence (9), has been shown to be an additional co-receptor during cellular infection of several HIV-1 strains (10).

The common polymorphism of CCR 2 is a $G \rightarrow A$ nucleotide transition at position 190 (counting from the ATG start codon), which results in the translocation of valine to isoleucine at position 64 of the CCR2 protein (11). Previously, CCR2V64I has been reported to be associated with delayed disease progression to AIDS. Patients with this V64I polymorphism developed full-blown AIDS approximately 2-4 years later than homozygous individuals carrying the wild-type allele (11-15). To date, some studies have suggested that CCR2-V64I is associated with a reduced risk of acquiring HIV-1 infection (16-21), while others suggest the opposite (22-29).

To elucidate the role of the CCR2-V64I polymorphism in HIV-1 infection, we performed a meta-analysis of all eligible related studies to obtain a decisive resolution, which may aid in understanding the level of risk of HIV-1 infection.

\section{Materials and methods}

Search strategy. Articles dated up to April 2010 were recovered from the EMBASE and MEDLINE databases via the PubMed search engine using the key words 'CCR2', 'polymorphism' and 'HIV' in various combinations. The references of the retrieved articles were perused to identify additional related studies. The search was limited to articles published in English.

Selection criteria. The following selection criteria were used to determine which studies were eligible: i) studies about the CCR2-V64I polymorphism and the risk of HIV-1 infection; ii) studies defined as case-control studies; iii) studies containing enough data; iv) studies in which genotype frequency data were available for both cases and controls. 
Data extraction. The following information was extracted from the eligible studies: first author, year of publication, country of study population, ethnicity, sample size, number of HIV-1-exposed, but uninfected, individuals, and distribution of CCR2 genotypes in cases and controls.

Statistical analysis. The primary assessment of effect was estimated by the odds ratio (OR) and associated $95 \%$ confidence interval $(\mathrm{CI})$. The pooled ORs were respectively calculated in four genetic models: a homozygote comparison (Ile/Ile vs. $\mathrm{Val} / \mathrm{Val}$ ), a heterozygote comparison (Ile/Ile vs. Val/Ile), a dominant model (Ile/Ile vs. Val/Val + Val/Ile) and a recessive model (Ile/Ile + Val/Ile vs. Val/Val). Stratified analysis was performed according to sample size, control population and ethnicity. Heterogeneity was assessed by a $\chi^{2}$-based Q-statistic test (30), and was considered significant if the P-value was $<0.10$. In such cases, the ORs were pooled according to the random-effects model (the DerSimonian and Laird model) (31); otherwise the fixed-effects model (the Mantel and Haenszel model) was used (32). Sensitivity analyses were conducted to evaluate the stability of the results. Analysis of publication bias was conducted with the funnel plot and Egger's linear regression asymmetry test, with a P-value of $<0.05$ taken to indicate a statistically significant publication bias. All statistical tests were conducted with STATA version 10.0 (Stata Corporation, College Station, TX, USA).

\section{Results}

Through a search of literature, 298 relevant articles were identified, 12 of which were determined to be eligible for further analysis (Fig. 1). The 12 studies included five populations of Asian descent, four mixed populations including participants of European, African or Asian descent and/or other ethnicities, two studies involving African subjects, and one including individuals of Caucasian descent. The total number of individuals analyzed was 6,599 (3,860 HIV-infected patients and 2,739 healthy controls). Six studies involved a total of 1,204 HIV-1-exposed, but uninfected, individuals. One study was performed on infants. The 12 eligible studies are summarized in Table I.

The distribution of the CCR2-V64I genotype among the study subjects is shown in Table II. Notably, some studies had found a relatively high frequency of the CCR2-V64I allele, ranging from 0.166 to $0.279(21,25,26)$.

After pooling the data from the 12 studies for meta-analysis, the results were calculated in a homozygote comparison ( $\mathrm{OR}=1.10,95 \%$ CI $0.79-1.53, \mathrm{P}=0.908$ for heterogeneity) (Fig. 2A), a heterozygote comparison (OR=0.98, 95\% CI 0.70 1.37, $\mathrm{P}=0.637$ for heterogeneity) (Fig. 2B), a dominant model ( $\mathrm{OR}=1.06,95 \%$ CI $0.77-1.47, \mathrm{P}=0.915$ for heterogeneity) (Fig. 2C) and a recessive model (OR=0.98, 95\% CI 0.77-1.27, $\mathrm{P}=0.000$ for heterogeneity, by random effects model) (Fig. 2D).

Stratified analysis was further performed to adjust for the effect of heterogeneity and to illustrate the exact relationship between the CCR2-V64I polymorphism and susceptibility to HIV-1 infection. The studies were divided into three subgroups according to control population: one subgroup of high-risk controls including 1,204 exposed, but uninfected, individuals and 3,017 HIV-1-infected patients; one subgroup of unexposed

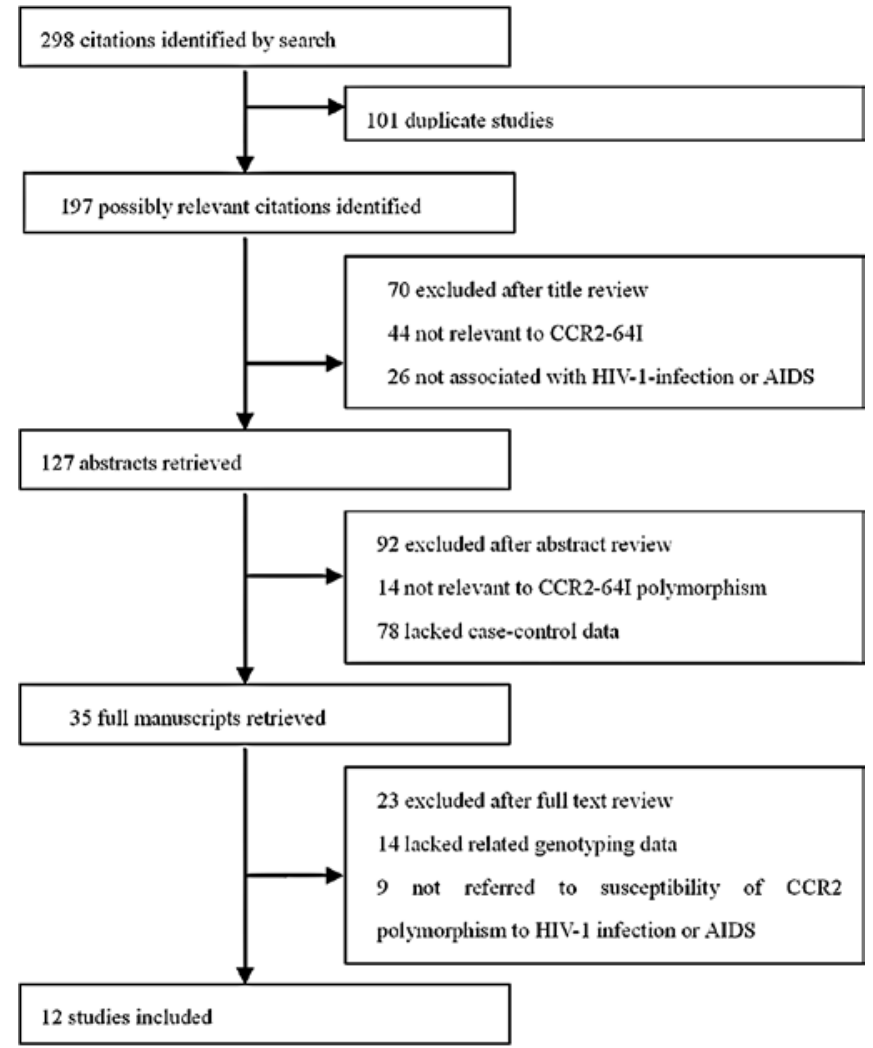

Figure 1. Flow diagram of study searching and selection process.

controls, including 2,754 unexposed individuals and 1,164 HIV-1-infected patients; and a final subgroup comprising patients from only one study (26), defined as a mother-to-child transmission subgroup. No evidence of a significant association between the CCR2-V64I polymorphism and susceptibility to HIV-1 infection was found in any genetic model among these subgroups (Table III). The studies were also stratified into two subgroups according to sample size: one comprising studies with more than 300 subjects; the other, studies with fewer than 300 subjects. In these subgroups, no apparant evidence of a significant association between the variant CCR2-V-64I genotype and the risk of HIV-1 infection was found (Table III). The studies were finally divided into four subgroups according to ethnicity. After pooling the data, no evidence of an association between the CCR2-V64I polymorphism and susceptibility to HIV-1 infection was found among Asian, African or Caucasian populations in any genetic model. In the mixed populations subgroup, a significantly decreased risk of HIV-1 was found in the heterozygote comparison $(\mathrm{OR}=0.33$, 95\% CI $0.11-0.98, \mathrm{P}=0.352$ for heterogeneity). However, no similar result was found in the homozygote comparison, the dominant model or the recessive model (Table III).

Sensitivity analysis was performed for various comparisons in the total population and all the subgroups. When one study (21) was excluded, the results became statistically significant $(\mathrm{OR}=0.61,95 \% \mathrm{CI} 0.41-0.91, \mathrm{P}=0.196$ for heterogeneity) in a recessive model among the subgroup of fewer than 300 subjects. However, the result for the recessive model in the mixed population remained insignificant (data not shown), and when another study (28) was excluded, the results in the recessive model among the Caucasian population were 
Table I. Characteristics of included studies.

\begin{tabular}{|c|c|c|c|c|c|}
\hline $\begin{array}{l}\text { First author } \\
\text { (reference) }\end{array}$ & Year & Country & Ethnicity & Sample size ${ }^{a}$ & $\begin{array}{l}\text { Sample size of high-risk } \\
\text { groups }^{\text {b }} \text { (case/control) }\end{array}$ \\
\hline Roman & 2002 & Luxembourg & Mixed $^{c}$ & $257 / 158$ & ND \\
\hline Wang & 2003 & China & Asian & $330 / 474$ & 474 \\
\hline Philpott & 2004 & USA & Mixed $^{c}$ & $1940 / 513$ & 283 \\
\hline Paz-y-Mino & 2005 & Ecuador & Mixed $^{c}$ & $50 / 222$ & ND \\
\hline Brouwer & 2005 & Kenya & African & $78 / 267^{\mathrm{d}}$ & ND \\
\hline Carvalhaes & 2005 & Brazil & Mixed $^{c}$ & $110 / 112$ & ND \\
\hline Suresh & 2006 & India & Asian & $49 / 110$ & 35 \\
\hline Shrestha & 2006 & USA & African & $100 / 100$ & 100 \\
\hline Kaur & 2007 & India & Asian & $180 / 221$ & ND \\
\hline Wichukchinda & 2008 & Thailand & Asian & $347 / 74$ & 74 \\
\hline Parczewski & 2009 & Poland & Caucasian & $168 / 150$ & ND \\
\hline Tan & 2010 & China & Asian & $251 / 238$ & 238 \\
\hline
\end{tabular}

${ }^{a}$ Only individuals for which genotype data was obtained. ${ }^{b} \mathrm{HIV}$-1-exposed, but uninfected, individuals. ${ }^{\mathrm{c} I n d i v i d u a l s ~ o f ~ E u r o p e a n, ~ A f r i c a n, ~ A s i a n ~}$ and/or other descent. 'Infants. ND, no data.

Table II. Distribution of CCR2-Val64Ile genotype among HIV-1-infected cases and controls.

\begin{tabular}{|c|c|c|c|c|c|c|c|c|c|c|c|c|}
\hline \multirow[t]{3}{*}{ Author } & \multicolumn{6}{|c|}{ Cases } & \multicolumn{6}{|c|}{ Controls } \\
\hline & \multicolumn{2}{|c|}{ Val/Val } & \multicolumn{2}{|c|}{ Val/Ile } & \multicolumn{2}{|c|}{ Ile/Ile } & \multicolumn{2}{|c|}{ Val/Val } & \multicolumn{2}{|c|}{ Val/Ile } & \multicolumn{2}{|c|}{ Ile/Ile } \\
\hline & No. & $\%$ & No. & $\%$ & No. & $\%$ & No. & $\%$ & No. & $\%$ & No. & $\%$ \\
\hline Roman & 219 & 85.2 & 38 & 14.8 & 0 & 0.0 & 128 & 81.0 & 30 & 18.9 & 0 & 0.0 \\
\hline Wang & 221 & 67.0 & 83 & 25.2 & 26 & 7.9 & 315 & 66.5 & 130 & 27.4 & 29 & 6.1 \\
\hline Philpott & 1,670 & 86.1 & 251 & 12.9 & 19 & 1.0 & 445 & 86.7 & 61 & 11.9 & 7 & 1.4 \\
\hline Paz-y-Mino & 20 & 40.0 & 28 & 56.0 & 2 & 4.0 & 169 & 76.0 & 40 & 18.0 & 13 & 6.0 \\
\hline Brouwer & 42 & 53.8 & 32 & 41.0 & 4 & 5.1 & 191 & 52.0 & 160 & 43.6 & 16 & 4.4 \\
\hline Carvalhaes & 98 & 89.1 & 12 & 10.9 & 0 & 0.0 & 84 & 75.0 & 28 & 25.0 & 0 & 0.0 \\
\hline Suresh & 36 & 72.0 & 12 & 24.0 & 1 & 2.0 & 80 & 72.7 & 27 & 24.5 & 3 & 2.7 \\
\hline Shrestha & 78 & 78.0 & 21 & 21.0 & 1 & 1.0 & 70 & 70.0 & 28 & 28.0 & 2 & 2.0 \\
\hline Kaur & 144 & 80.0 & 32 & 17.7 & 4 & 2.2 & 173 & 78.2 & 44 & 19.9 & 4 & 1.8 \\
\hline Wichukchinda & 239 & 68.9 & 95 & 27.4 & 13 & 3.7 & 49 & 66.2 & 21 & 28.4 & 4 & 5.4 \\
\hline Parczewski & 136 & 81.0 & 31 & 18.5 & 1 & 0.6 & 117 & 78.0 & 31 & 42.8 & 2 & 1.3 \\
\hline Tan & 130 & 51.8 & 102 & 40.6 & 19 & 7.6 & 140 & 58.8 & 85 & 35.7 & 13 & 5.5 \\
\hline
\end{tabular}

also not statistically significant (data not shown). In other subgroups, sensitivity analysis revealed no significant results (data not shown).

Publication bias was assessed by Begg's funnel plot and Egger's test (data not shown) in the total population and all the subgroups. Egger's linear regression test suggested a significant publication bias in the dominant model (II vs. VV + VI) for total population $(\mathrm{t}=-2.73, \mathrm{P}=0.026)$, but no evidence of publication bias in any other genetic model for the total population or subgroups (data not shown).

\section{Discussion}

In order to elucidate the association between the CCR2-V64I polymorphism and susceptibility to HIV-1 infection, we performed a meta-analysis of data from 12 eligible studies including a total of 3,860 HIV-infected patients and 2,739 controls. The results indicate that the CCR2-V64I polymorphism has no effect on the risk of HIV-1 infection among worldwide populations in any genetic model, consistent with the results of certain earlier studies (22-29). Taking into account 
A

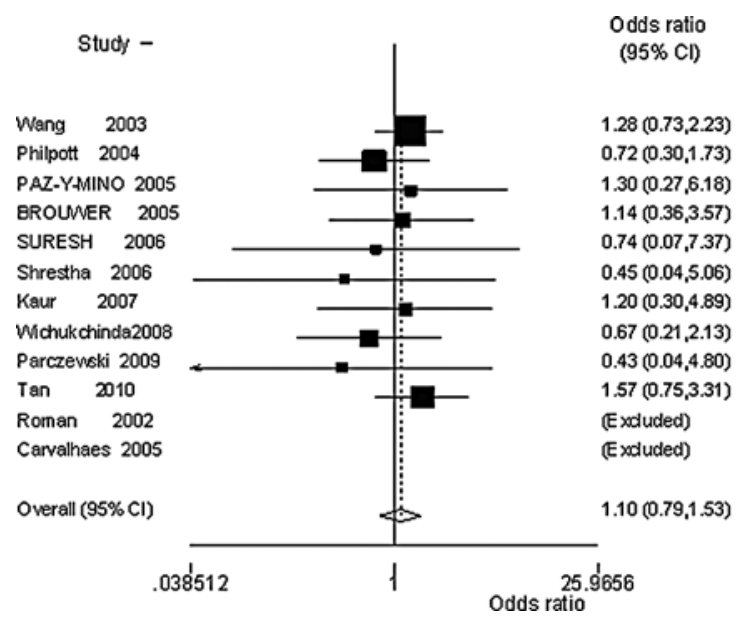

C

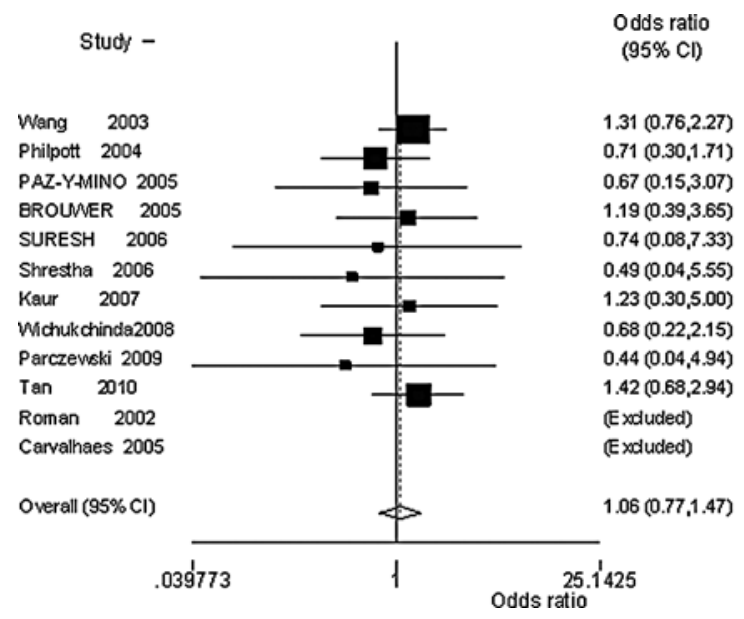

\begin{tabular}{|c|} 
\% Weight \\
\\
32.1 \\
16.2 \\
3.8 \\
7.9 \\
2.7 \\
3.1 \\
5.3 \\
9.3 \\
3.1 \\
16.6 \\
0.0 \\
0.0 \\
\end{tabular}

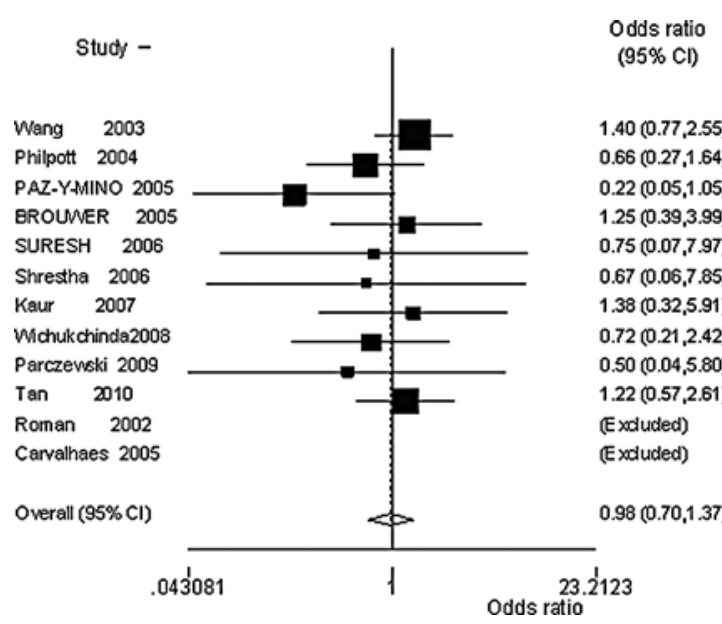

D

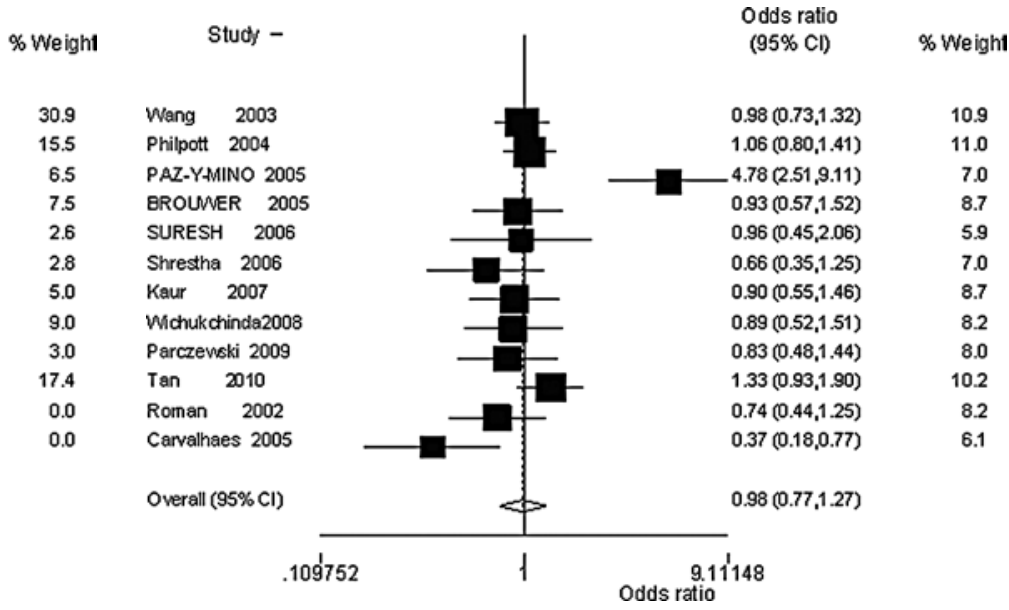

Figure 2. Meta-analysis of the relationship between the CCR2-V64I polymorphism and HIV-1 infection using (A) a homozygote comparison, (B) a heterozygote comparison, (C) a dominant model, and (D) a recessive model.

the effect of ethnicity, sample size and control population, stratified analyses were performed. The results obtained for the various subgroups did not point to an association between the CCR2-V64I polymorphism and HIV-1 infection, with one exception: populations of mixed ethnicity.

Among populations of mixed ethnicity, the CCR2-64II genotype was associated with a reduced risk of HIV-1 infection as compared to the CCR2-64VI genotype. This suggests that the association between the CCR2-V64I polymorphism and susceptibility to HIV-1 infection varies between ethnic populations. One study reported a higher frequency of CCR2-V64I genotypes in Chinese individuals relative to Caucasians (19). Other studies showed a higher frequency of the CCR2-V64I genotype among African populations relative to populations of European descent $(5,11)$. Nevertheless, no significant association was found between the CCR2-V64I polymorphism and susceptibility to HIV-1 infection in analyses of the total population. However, among the mixed ethnic population, there were relatively few people recruited to each investigation. In conclusion, the protective effect of
CCR2-V64I on HIV-1 infection that we observed may have been due to different ethnic backgrounds and small sample size. We conclude that further large-scale analysis is required.

The entry of HIV into the CD4 lymphocyte is dependant on the binding of necessary co-receptors (4). Certain chemokine receptors have been demonstrated to play critical roles in HIV-1 infection (3). Some polymorphisms or mutations may have effects on the ability of the virus to infect cells, influencing HIV-1 infectivity (29). CCR2, which acts as a co-receptor mediating infection of some strains of HIV-1, has been found to modulate the susceptibility to HIV-1 of individuals carrying a mutation at position 64 (11). However, our present analysis found that the CCR2-V64I polymorphism has no effect on susceptibility to HIV-1 infection. According to several studies, the CCR2-64I protein preferentially dimerizes with CXCR4 polypeptides to affect HIV-1 entry and delay disease progression to AIDS (11-14). It is possible that the CCR2-V64I polymorphism is more closely associated with slower progression to AIDS than with susceptibility to HIV-1 infection. 


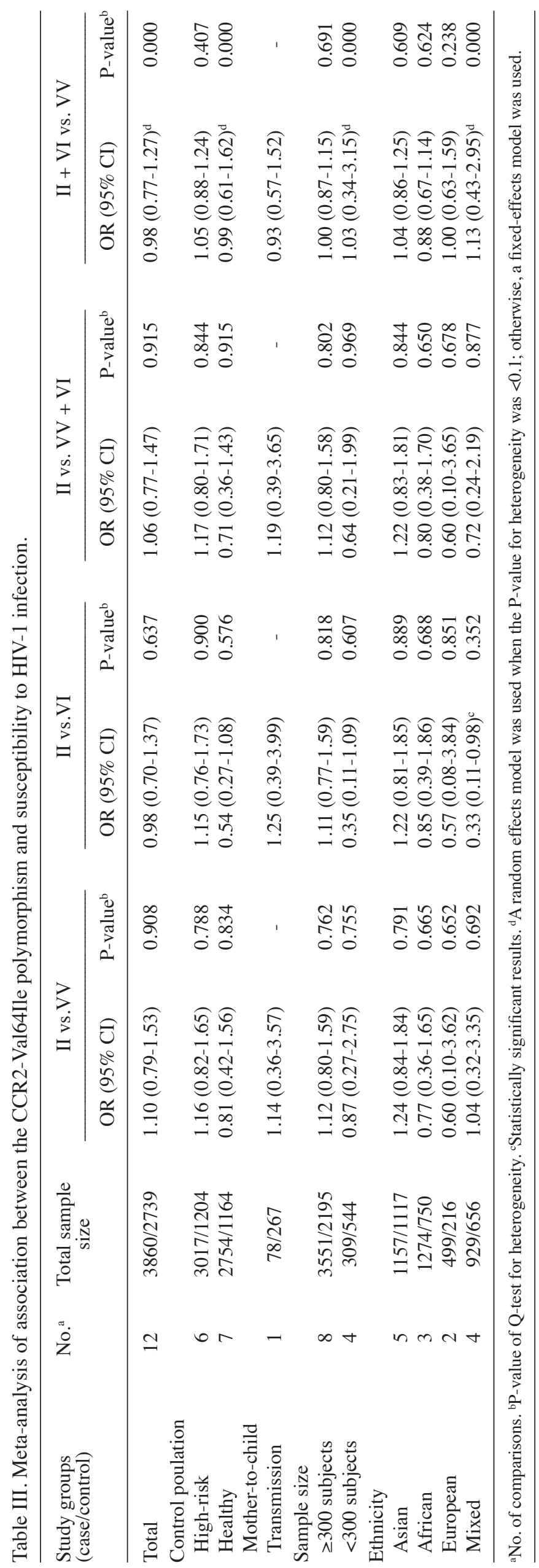

Although meta-analysis is a powerful statistical method, it has certain limitations. In the present study, a publication bias existed in a dominant model among the total population. Therefore, more studies with larger sample sizes are required. Additionally, sensitivity was detected in a recessive model among some subgroups.

In conclusion, our results suggest that the CCR2-V64I polymorphism has no effect on reducing the risk of HIV-1 infection. Since the distribution of genotypes varies according to race and ethnicity, studies on more subjects recruited from specific regions worldwide are required.

\section{Acknowledgements}

We gratefully acknowledge Lei Yao at Fudan University for help. This study was supported by the National Grand Program on Key Infectious Disease (2008ZX10001-006), the National Natural Science Foundation of China (no. 30972609), and the Key Basic Research Program of Shanghai (no. 08JC1401300).

\section{References}

1. Singh P, Kaur G, Sharma G and Mehra NK: Immunogenetic basis of HIV-1 infection, transmission and disease progression. Vaccine 26: 2966-2980, 2008.

2. Dean M, Carrington M, Winkler C, et al: Genetic restriction of HIV-1 infection and progression to AIDS by a deletion allele of the CCR5 structural gene. Science 273: 1856-1862, 1996.

3. Fauci AS: Resistance to HIV-1 infection: it's in the genes. Nat Med 2: 966-967, 1996

4. Paxton WA and Kang S: Chemokine receptor allelic polymorphisms: relationships to HIV resistance and disease progression. Semin Immunol 10: 187-194, 1998.

5. Mummidi S, Ahuja SS, Gonzalez E, Anderson SA, Santiago EN, Setphan KT, Craig FE, O'Connell P, Tryon V, Clark RA, Dolan MJ and Ahuja SK: Genealogy of the CCR5 locus and chemokine system genevariants associated with altered rates of HIV-1 disease progression. Nat Med 4: 786-793, 1998.

6. Michael NL: Host genetic influences on HIV-1 pathogenesis. Curr Opin Immunol 11: 466-474, 1999.

7. McDermott DH, Beecroft MJ, Kleeberger CA, Al-Sharif FM, Ollier WE, Zimmerman PA, Boatin BA, Leitman SF, Detels R, Hajeer AH and Murphy PM: Chemokine RANTES promoter polymorphism affects risk of both HIV infection and disease progression in the Multicenter AIDS Cohort Study. AIDS 14: 2671-2678, 2000.

8. Singh KK, Barroga CF, Hughes MD, Chen J, Raskino C, McKinney RE and Spector SA: Genetic influence of CCR5, CCR2, and SDF1 variants on human immunodeficiency virus 1 (HIV-1)-related disease progression and neurological impairment, in children with symptomatic HIV-1 infection. J Infect Dis 188: 1461-1472, 2003.

9. Daugherty BL and Springer MS: The $\beta$-chemokine receptor genes CCR1 (CMKBR1), CCR2 (CMKBR2), and CCR3 (CMBRK3) cluster within $285 \mathrm{~kb}$ on human chromosome3p21. Genomics 41: 294-297, 1997.

10. Doranz BJ, Rucker J, Yi Y, Smyth RJ, Samson M, Peiper SC, Parmentier M, Collman RG and Doms RW: A dual-tropic primary HIV-1 isolate that uses fusin and the $\beta$-chemokine receptors CKR-5, CKR-3, and CKR-2b as fusin cofactors. Cell 85: 1149-1158, 1996.

11. Smith MW, Dean M, Carrington M, et al: Contrasting genetic influence of CCR2 and CCR5 variants on HIV-1 infection and disease progression. Science 277: 959-965, 1997.

12. Kostrikis LG, Huang Y, Moore JP, Wolinsky SM, Zhang L, Guo Y, Deutsch L, Phair J, Neumann AU and Ho DD: A chemokine receptor CCR2 allele delays HIV-1 disease progression and is associated with a CCR5 promoter mutation. Nat Med 4: 350-353, 1998.

13. Ioannidis JP, Contopoulos-Ioannidis DG, Rosenberg PS, et al: Effects of CCR5-delta32 and CCR2-64I alleles on disease progression of perinatally HIV-1-infected children: an international meta-analysis. AIDS 17: 1631-1638, 2003. 
14. Mellado M, Rodriguez-Frade JM, Vila-Coro AJ, de Ana AM and Martinez AC: Chemokine control of HIV-1 infection. Nature 400: 723, 1999.

15. Ioannidis JP, Rosenberg PS, Goedert JJ, et al: Effects of CCR5delta32, CCR2-64I, and SDF-1 3'A alleles on HIV-1 disease progression: an international meta-analysis of individual-patient data. Ann Intern Med 135: 782-795, 2001.

16. Louisirirotchanakul S, Liu H, Roongpisuthipong A, Nakayama EE, Takebe Y, Shioda T and Wasi C: Genetic analysis of HIV-1 discordant couples in Thailand: association of CCR2 64I homozygosity with HIV-1-negative status. J Acquir Immune Defic Syndr 29: 314-315, 2002.

17. Petersen DC, Laten A, Zeier MD, Grimwood A, Rensburg EJ and Hayes VM: Novel mutations and SNPs identified in CCR2 using a new comprehensive denaturing gradient gel electrophoresis assay. Hum Mutat 20: 253-259, 2002.

18. De PinhoLott Carvalhaes FA, Lemos Cardoso G, Rosário Vallinoto AC, et al: Frequencies of CCR5- $\Delta 32$, CCR2-64I and SDF1-3'A mutations in human immunodeficiency virus (HIV) seropositive subjects and seronegative individuals from the state of Pará in Brazilian Amazonia. Genet Mol Biol 28: 665-669, 2005.

19. Shrestha S, Strathdee SA, Galai N, et al: Behavioral risk exposure and host genetics of susceptibility to HIV-1 infection. J Infect Dis 193: 16-26, 2006.

20. Wichukchinda N, Nakayama EE, Rojanawiwat A, et al: Effects of CCR2 and CCR5 polymorphisms on HIV-1 infection in Thai females. J Acquir Immune Defic Syndr 47: 293-297, 2008.

21. Tan XH, Zhang JY, Di CH, et al: Distribution of CCR5- $\triangle 32$, CCR5 m303A, CCR2-64I and SDF1-3'A in HIV-1 infected and uninfected high-risk Uighurs in Xinjiang, China. Infect Genet Evol 10: 268-272, 2010

22. Roman F, Franck N, Burgy C, et al: Prevalence of HIV co-receptor polymorphisms in HIV-infected patients and uninfected volunteers in Luxembourg. HIV Clin Trials 3: 195-201, 2002.

23. Wang FS, Hong WG, Cao Y, et al: Population survey of CCR $5 \Delta 32$, CCR5m303, CCR2b64I, and SDF1 3'A allele frequencies in indigenous Chinese healthy individuals, and in HIV-1-infected and HIV-1-uninfected individuals in HIV-1 risk groups. J Acquir Immune Defic Syndr 32: 124-130, 2003.
24. Philpott S, Burger H, Tarwater PM, et al: CCR2 genotype and disease progression in a treated population of HIV type 1-infected women. Clin Infect Dis 39: 861-865, 2004.

25. Paz-y-mino C, Morillo SA, Celi AP, et al: CCR5 32, CCR2-64I, and SDF1-3'A polymorphisms related to resistance to HIV-1 infection and disease in the Ecuadorian population. Human Biology 77: 521-526, 2005.

26. Brouwer KC, Yang CF, Parekh S, et al: Effect of CCR2 chemokine receptor polymorphism on HIV type 1 mother-to-child transmission and child survival in Western Kenya. AIDS Res Hum Retrov 21: 358-362, 2005

27. Suresh P, Wanchu A, Kaur Sachdeva R and Bhatnagar A: Gene polymorphisms in CCR 5, CCR 2, CX3CR1, SDF-1 and RANTES in exposed but uninfected partners of HIV-1 infected individuals in North India. J Clin Immunol 26: 476-484, 2006.

28. Kaur G, Singh P, Kumar N, Rapthap CC, Sharma G, Vajpayee M, Wig N, Sharma SK and Mehra NK: Distribution of CCR2 polymorphism in HIV-1-infected and healthy subjects in North India. Int J Immunogenet 34: 153-156, 2007.

29. Parczewski M, Leszczyszyn-Pynka M, Kaczmarczyk M, Adler G Binczak-Kuleta A, Loniewska B, Boroñ-Kaczmarska A and Ciechanowicz A: Sequence variants of chemokine receptor genes and susceptibility to HIV-1 infection. J Appl Genet 50: 159-166, 2009.

30. Cochran WG: The combination of estimates from different experiments. Biometrics 10: 29, 1954.

31. DerSimonian R and Laird N: Meta-analysis in clinical trials. Control Clin Trials 7: 177-188, 1986.

32. Mantel N and Haenszel W: Statistical aspects of the analysis of data from retrospective studies of disease. J Natl Cancer Inst 22: 719-748, 1959. 